\title{
Fractal analysis of otolith microrelief as a method for determines relationship of species
}

\author{
M.L. Tyagun ${ }^{1 *}$, A.A. Golovko $2 * *$ \\ ${ }^{1}$ Limnological Institute SB RAS, Irkutsk, Russia \\ ${ }^{2}$ Institute of Solar-Terrestrial Physics SB RAS, Irkutsk, Russia \\ *mary@lin.irk.ru,**golovko@iszf.irk.ru
}

Key words: crystalline surface, otolith, fractal analysis, multifractal spectrum

The micromorphology of fish otolith (texture diagnostics) in recent years has attracted interest from specialists in various fields. The mechanisms of the organization of the microstructural tissue and its functional features are not clear. On the otolith, in an important functional part of the acoustic apparatus of fish, a hierarchy of crystallized elements is formed in the zone of the sulcus acousticus. A specialized neural tissue, the auditory macula, adjoins a macular spot containing similar elements. The macula consists of sensory cells interacting with the surface of the microstructure. Acoustic vibrations of the otolith cause mechanical action and polarization of the cells. Structural organization of the macula is usually presented in the form of a simple scheme consisting of regions that symbolize groups of cells of different lengths, with different polarization vectors. It is considered that a polarized macular pattern is identical to the otolith macular pattern including areas with structures of different types as well. Each area is visualized as hierarchic levels of multidimensional geometrical objects. Therefore, to concept its organization, a scheme similar to the sensory macula is not enough. Conception the principle of structural organization of tissue of this kind is possible with the help of fractal analysis, capable of describing the nature of the geometry of a complex object. Microcanonical analysis of the multifractal spectra confirmed the fractal origin of the hierarchic organization of the crystallized otolith surface. Multifractal pattern of this biomineral surface is consistent with the ideas on these structures formation in nature (insentient substance). Instantaneous temporal analysis of the complex self-organizing processes in nature, such as lithospheric orogeny or fluid flow through porous media showed their fractal geometry. It seems that regulation of the otolith microrelief formation by the organism is facilitated by sustaining non-isotropy of its tissue that is required for fixation of the arriving signal by the acoustic organs and space orientation. The similarity of multifractal spectral patterns of stone sculpins ( $P$. knerii), described for different areas of crystallized surface using different scales, was found. Thus, we are convinced that the scale variations during analysis do not affect the quality of the data obtained. The concave curves of the multiscale images of the same area of stone sculpins are located within the same range of values and reach the same level. The multifractal spectra of the crystalline patterns of four species selected for analysis is differ. This supports a hypothesis on the unique species organization of the crystallized surface of the biomineral. Based upon the species spectral curves integrated into pairs by their relationship distance, we conclude that the closer is the relationship, the greater is the number of common features of the external microreliefs (stone and sand sculpins, P. knerii, L. kesslerii) and the larger is the taxonomical divergence (grayling and dace, T. baicalensis, L. leuciscus), the greater are the differences in the multifractal spectra. The data obtained allow us to state that the fractal analysis provides a possibility to describe the organization of crystalline elements of the biomineral and use its fractal parameters, multifractal spectrum in particular, for taxonomic analysis. 